prevention program for core and bridge population groups across 4 Southern and 2 Northeastern states in India representing approximately $80 \%$ of India's HIV burden in 2002. Avahan offered a standardised package of known and proven prevention interventions to high-risk groups and bridge populations in the geographic areas most affected by the epidemic. The program reached 220000 female sex workers; 80000 High risk MSM; 18000 Injecting drug users and 5 million men at risk. By December 2008, Avahan had achieved significant scale and coverage with over $75 \%$ of total denominator including FSW and high risk MSM contacted monthly through outreach. The presentation will include the challenges at the start of the program, Avahan approaches to rapid scale up while leveraging data to ensure focus on highest at risk through provision of a standardised package of services (known as the Common Minimum Program).

\section{S7.2 MEASUREMENT AND COMMUNITY MOBILISATION DO NOT HAVE TO BE MUTUALLY EXCLUSIVE: PRELIMINARY ANALYSIS FROM A SCALED PROGRAMIME}

doi:10.1136/sextrans-2011-050102.28

\section{T Wheeler. Bill \& Melinda Gates Foundation}

Community mobilisation approaches within the HIV/AIDS landscape have rarely operated at a significant scale, or been designed with the conceptual and technical clarity necessary to be measured effectively. Measurement of community mobilisation by necessity has been undertaken through innovative qualitative approaches but these have often lacked the rigour or complementary qualitative methods to provide adequate analysis on the association with outcomes or the path to generating HIV/AIDS outcomes. Avahan may provide new insights into community mobilisation and structural interventions as an effective and replicable approach within comprehensive HIV prevention programming. The experience of retrofitting measurement into a "living" programme and developing measurement indicators and tools to work at scale provide practical examples of what can be done. The Avahan logic model, proposed measurement approach and some preliminary analysis of risk reduction and sustainability outcomes as a result of community mobilisation and structural interventions will be presented.

\section{S7.3 IMPACT OF THE AVAHAN INTERVENTION ON HIV/STI TRANSIMISSION AMONGST HIGH AND LOW-RISK GROUPS: AN INTERIM MODELLING ASSESSMENT}

\section{doi:10.1136/sextrans-2011-050102.29}

${ }^{1} \mathrm{P}$ Vickerman, ${ }^{1,2} \mathrm{M}$ Pickles, ${ }^{1,3} \mathrm{C} \mathrm{M}$ Lowndes, ${ }^{4,5} \mathrm{~B}$ M Ramesh, ${ }^{4,6} \mathrm{R}$ Washington, ${ }^{5} \mathrm{~S}$ Moses, ${ }^{7} \mathrm{~K}$ Deering, ${ }^{5} \mathrm{~S}$ Reza-Paul, ${ }^{1} \mathrm{~A}$ Vassall, ${ }^{8,9} \mathrm{~J}$ Bradley, ${ }^{5} \mathrm{~J}$ Blanchard, ${ }^{9} \mathrm{M}$ Alary, ${ }^{2,9} \mathrm{M}$ C Boily. ${ }^{1}$ London School of Hygiene \& Tropical Medicine, UK; ${ }^{2}$ Imperial College, London, UK; ${ }^{3}$ Health Protection Agency, London, UK; ${ }^{4}$ Karnataka Health Promotion Trust, Bangalore, India; ${ }^{5}$ University of Manitoba, Winnipeg, Canada; ${ }^{6}$ St. John's Medical College and Hospital, Bangalore, India; 'University of British Columbia, Vancouver, Canada; ${ }^{8}$ CHARME-India Project, Bangalore, India; ${ }^{9}$ URESP, Centre de recherche FRSO du CHA universitaire de Québec, Québec, Canada

Objective To estimate the potential HIV-impact of Avahan, the India AIDS Initiative, among targeted high-risk groups (including female sex workers (FSWs), their clients and men who have sex with men (MSM)) and the general population in different districts of Karnataka, Andhra Pradesh, Tamil Nadu and Maharashtra.

Design Impact evaluation involving mathematical modelling using detailed serial cross-sectional surveys on sexual behaviour and STI/ HIV prevalence (IBBA) among targeted high-risk groups and the general population.
Methods A bespoke detailed deterministic model, parameterised with district specific IBBA data, was used to simulate HIV/HSV-2/ syphilis transmission in high-risk groups and the general population in different districts. Latin hypercube sampling within a Bayesian framework was used to identify multiple parameter sets that reproduced multiple rounds of HIV prevalence data among FSWs, MSM and clients in all districts and the general population for some districts. The framework was used to test which of two hypotheses (H1 and H2) for time trends in consistent condom use (CCU)

\section{A) Mysore}

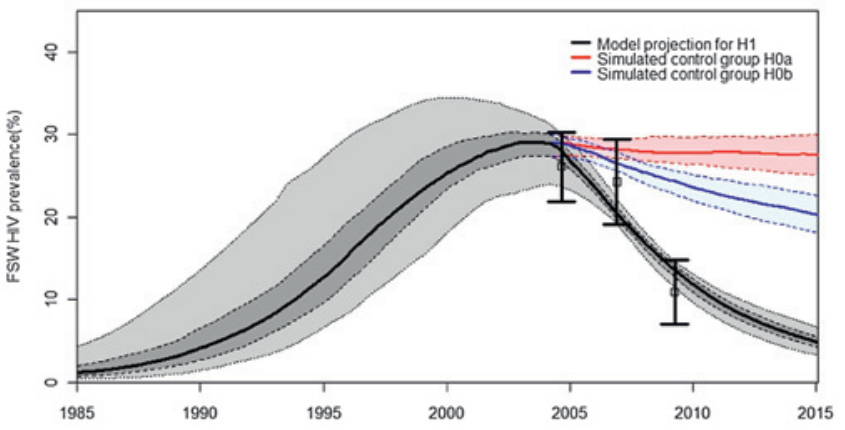

\section{B) Belgaum}

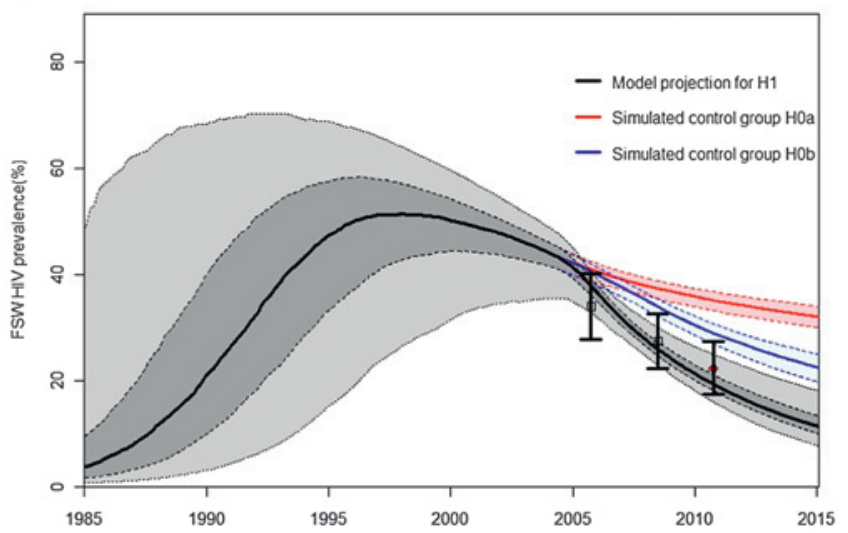

C) Bellary

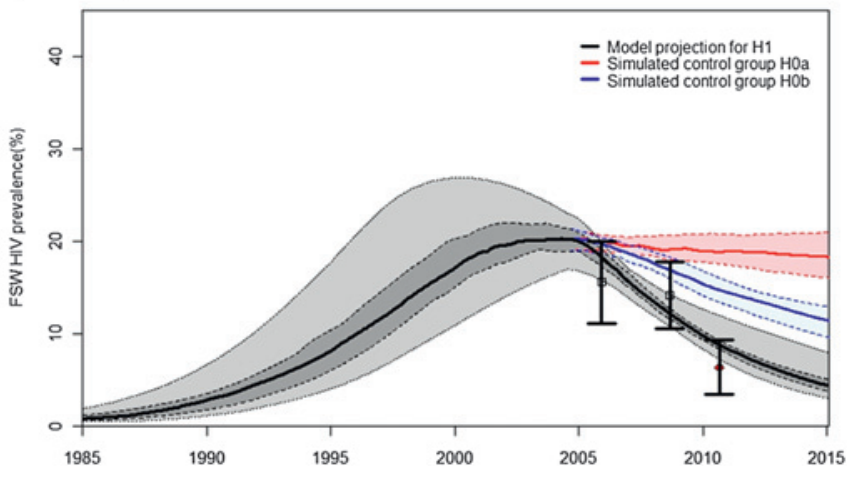

Abstract S7.3 Figure 1 Predicted FSW HIV prevalence over time for the most likely hypothesis $(\mathrm{H} 1)$ and the two null hypotheses $\mathrm{HOa}$ and $\mathrm{HOb}$ used to simulate control groups (constant or slowly increasing condom use since start of Avahan) in A) Mysore, B) Belgaum, and C) Bellary districts. Shown on the graphs are the mean (dark lines black, blue and red) and the $75 \%$ credibility intervals (shaded area) for each hypothesis. The paler grey area represents the $95 \%$ credibility intervals. Also shown is the available IBBA survey prevalence data (mean and $95 \% \mathrm{CI}$ ). 